\title{
SHARP CONSTANT IN NONLOCAL INEQUALITY AND ITS APPLICATIONS TO NONLOCAL SCHRÖDINGER EQUATION WITH HARMONIC POTENTIAL*
}

\author{
JIANQING $\mathrm{CHEN}^{\dagger}$, BOLING GUO $\ddagger$, AND YONGQIAN HAN ${ }^{\S}$
}

Abstract. This paper contains two parts. In the first part, we derive a variant of GagliardoNirenberg interpolation inequality involving nonlocal nonlinearity and determine its best (smallest) constant. In the second part, we study two applications of this inequality and its best constant. In the first application, we use this best constant to establish a sharp criterion for the global existence and blow-up of solutions of the inhomogeneous Schrödinger equation with harmonic potential and nonlocal nonlinearity

$$
i \varphi_{t}=-\triangle \varphi+|x|^{2} \varphi-\varphi|\varphi|^{p-2} \int \frac{|\varphi(y)|^{p}}{|x-y|^{\alpha}} d y
$$

in the critical case $p=2+(2-\alpha) / N$. The result indicates that the existence of blow-up solutions not only depends on the mass of the initial data but also on the profile of the initial data. In the second application, we use this best constant to prove that when $2+(2-\alpha) / N<p<(2 N-\alpha) /(N-2)$, the solutions exist globally in time for one class of initial data whose norm can be as large as one wants.

Key words. Nonlocal interpolation inequality, sharp constant, global solutions, blow-up, nonlocal Schrödinger equation.

2000 subject classifications. 35J20, 35Q55.

\section{Introduction}

This paper is concerned with the nonlinear Schrödinger equation with harmonic potential

$$
i \varphi_{t}+\triangle \varphi-|x|^{2} \varphi+F(\varphi)=0, \quad x \in \mathbb{R}^{N}, \quad t \geq 0,
$$

where $\varphi:=\varphi(x, t): \mathbb{R}^{N} \times \mathbb{R}_{+} \rightarrow \mathbb{C}$ is a complex-valued function and $F(\varphi)$ is a nonlinearity (possibly nonlocal) satisfying suitable assumptions given later.

Equation (1.1) models a lot of physical phenomena. For example it is known as the Gross-Pitaevskii (GP) equation in the context of Bose-Einstein condensates (BEC) with parabolic traps. In fact, assuming a highly anistropic trap, Kivshar et al [17] derived the GP-equation equation (1.1) with $F(\varphi)=\varphi|\varphi|^{2}$ as a model equation for the macroscopic dynamics of cooled atoms confined in a three dimensional parabolic potential created by a magnetic trap. Deconinck et al derive a three dimensional Schrödinger equation with potential and nonlocal nonlinearity; see [10, Equ. (5)].

Equation (1.1) with nonlocal nonlinearity $F(\varphi)$ has also appeared in other applications. For example, Kurth derived in [18] that Schrödinger equation with harmonic potential and nonlocal nonlinearity can be used to describe average pulse propagation

\footnotetext{
*Received: December 12, 2008; accepted (in revised version): April 2, 2009. Communicated by Jack Xin.

${ }^{\dagger}$ School of Mathematics and Computer Science, Fujian Normal University, Cangshan Campus, Fuzhou 350007, P.R. China (chenjq_iapcm@126.com; jqchen@fjnu.edu.cn).

J. Chen is supported by NSF of China (10501006), NSF of Fujian (2008J0189) and the program for NCETFJ.

${ }^{\ddagger}$ Institute of Applied Physics and Computational Mathematics, PO Box 8009, Beijing 100088, P.R. China.

$\S$ Institute of Applied Physics and Computational Mathematics, PO Box 8009, Beijing 100088, P.R. China.
} 
in dispersion-managed fibers. Usually the nonlocal nonlinearity is assumed to be of Hartree type, i.e.,

$$
F(\varphi)=\left(V *|\varphi|^{2}\right) \varphi,
$$

where the kernel $V$ is in some weak $L^{q}$-space and $*$ denotes convolution, see e.g. $[10,14]$ and the references therein. It is of interest to understand the original nonlocal problem directly, although in most applications it is hard to get a clue on properties of the kernel.

These motivate us to study equation (1.1) directly with nonlocal nonlinearity of the following form

$$
i \varphi_{t}=-\triangle \varphi+|x|^{2} \varphi-\varphi|\varphi|^{p-2} \int_{\mathbb{R}^{N}} \frac{|\varphi(y)|^{p}}{|x-y|^{\alpha}} d y, \quad t \geq 0, x \in \mathbb{R}^{N},
$$

where $p \geq 2$ and $0<\alpha<N$. The purpose here is to extend those results for the Schrödinger equation with local nonlinearity to the case of nonlocal nonlinearity and shed some new light for the Schrödinger with nonlocal nonlinearity. We remark that when $\alpha=1, p=2$ and $N=3$, equation (1.2) is equivalent to the Schrödinger-Possion system with harmonic potential,

$$
\left\{\begin{aligned}
i \varphi_{t}+\triangle \varphi & =|x|^{2} \varphi-V(x) \varphi, \quad x \in \mathbb{R}^{N} \\
\triangle V & =|\varphi|^{2} .
\end{aligned}\right.
$$

This equation typically arises in the mean field approximation of many body effects, modeled by the Possion equation with a confinement modeled by the quadratic potential of the harmonic oscillator. If the potential vanishes, then this equation models the classical limit of the field equations describing quantum mechnical non-relativistic many boson system [1]. For other variants of the Schrödinger equation, we refer the interested reader to $[6]$ and the references therein.

Now we come back to equation (1.2). Given an initial data

$$
\varphi(x, 0)=\varphi_{0}(x),
$$

we are interested in the following question: Under what conditions the solutions of equations (1.2)-(1.3) exist globally in time and under what conditions the solutions of equations (1.2)-(1.3) blow up in a finite time?

Noting that in the case of local nonlinearity $F(\varphi)=\varphi|\varphi|^{p-2}$, one can use the standard Gagliardo-Nirenberg inequality to study equation (1.1); we refer the interested readers to $[11,22,25,7,8]$ for the stability of standing waves as well as global existence of equation (1.1) with various initial data. However, for the nonlocal nonlinearity like equation (1.2), the issue of whether or not a particular choice of initial data generates a blow-up solution of equation (1.2) is more subtle. In particular the arguments used in [7] cannot deduce a sharp condition on the existence of global solutions or blow-up solutions of equation (1.2), so we need to find other ways as we will see in the present paper.

In section 2, we derive a variant of the Gagliardo-Nirenberg interpolation inequality involving nonlocal nonlinearity and determine the best (smallest) constant; see Theorem 2.3 and Theorem 2.7. We emphasize that the best constant determined here is not only of independent interest, but can also be used to study equations (1.2)-(1.3). In section 3, we sketch some results on the Cauchy problem of equation (1.2). In section 4 , since we deal with the problem harmonic potential $|x|^{2}$, we use 
Theorem 2.7 to establish a sharp criterion on the solutions of equations (1.2)-(1.3) in the critical nonlinearity $p=2+(2-\alpha) / N$; see Theorem 4.1 and Theorem 4.3. The results indicate a sharp threshold on the existence of global solutions of equation (1.2). Furthermore, the results suggest the existence of blow-up solutions of equation (1.2) depends on both the mass of the initial data and the profile of the initial data. In section 5 , we give a sufficient condition to the existence of global solutions of equation (1.2) in the case where $2+(2-\alpha) / N<p<(2 N-\alpha) /(N-2)$. The interesting aspect is that equation (1.2) possesses global solutions even when one takes the initial data as large as one wants; see Theorem 5.3. The final section is an appendix where we outline the proof of Proposition 3.1.

To establish the results in view, we base our approach on an idea originated from $[24,2]$. We emphasize that the use of Theorem 2.7 is essential in the proofs of Theorem 4.1, Theorem 4.3, and Theorem 5.3. We point out that Theorem 2.7 can also be used to establish a sharp threshold to the existence and nonexistence of standing waves of NLS-equation with nonlocal nonlinearity as well as their stability. The results will be published elsewhere.

Notations. Throughout this paper, $H^{1}\left(\mathbb{R}^{N}\right)$ denotes the standard Sobolev space with the standard norm. We denote the norm of the space $L^{q}\left(\mathbb{R}^{n}\right)$ by $\|\cdot\|_{L^{q}}, 1 \leq q \leq \infty$, and denote the integral $\int_{\mathbb{R}^{N}} d x$ simply by $\int$ unless stated otherwise. We also denote various positive constants by $C$ or $C_{j}$. $\bar{v}$ denotes the complex conjugate and Re denotes the real part.

\section{Best (smallest) constant}

The goal of this section is to derive a variant of the Gagliardo-Nirenberg interpolation inequality (see Theorem 2.3) and determine its best constant (see Theorem 2.7). This inequality and the best constant will play an essential role in what follows. Firstly, we state some lemmas.

LEMma 2.1. (Gagliardo-Nirenberg inequality, see e.g. [5]) Let $1<q<\frac{N+2}{N-2}$ when $N \geq 3$ and $1<q<+\infty$ when $N=1,2$. Then there is a positive constant $C$ depending on $N$ and $q$ such that for any $u \in H^{1}\left(\mathbb{R}^{N}\right)$,

$$
\int|u|^{q+1} \leq C\left(\int|\nabla u|^{2}\right)^{\frac{N(q-1)}{4}}\left(\int|u|^{2}\right)^{\frac{q+1}{2}-\frac{N(q-1)}{4}} .
$$

Lemma 2.2. [21, p. 31] Let $0<\beta<N$ and $f \in L^{q}\left(\mathbb{R}^{N}\right), h \in L^{r}\left(\mathbb{R}^{N}\right)$ with $\frac{1}{q}+\frac{1}{r}+\frac{\beta}{N}=2$ and $1<q, r<\infty$. Then

$$
\int_{\mathbb{R}^{N} \times \mathbb{R}^{N}} \frac{|f(x)||h(y)|}{|x-y|^{\beta}} d x d y \leq C(q, r, \beta, N)\|f\|_{L^{q}\left(\mathbb{R}^{N}\right)}\|h\|_{L^{r}\left(\mathbb{R}^{N}\right)}, \quad x, y \in \mathbb{R}^{N},
$$

where $C(q, r, \beta, N)$ is a positive constant depending on $q, r, \beta$, and $N$.

Next we use Lemma 2.1 and Lemma 2.2 to derive a variant of Gagliardo-Nirenberg interpolation inequality.

Theorem 2.3. Let $0<\alpha<N$ and $(2 N-\alpha) / N<p<2^{*}(\alpha)$, where $2^{*}(\alpha):=(2 N-$ $\alpha) /(N-2)$ when $N \geq 3$ and $(2 N-\alpha) / N<p<+\infty$ when $N=1$, 2. Then there is a positive constant $C(p, \alpha, N)$ depending on $p, \alpha$ and $N$ such that for any $u \in H^{1}\left(\mathbb{R}^{N}\right)$,

$$
\int_{\mathbb{R}^{N} \times \mathbb{R}^{N}} \frac{|u(x)|^{p}|u(y)|^{p}}{|x-y|^{\alpha}} d x d y \leq C(p, \alpha, N)\left(\int|\nabla u|^{2}\right)^{A}\left(\int|u|^{2}\right)^{B},
$$


where $A=\frac{N(p-2)+\alpha}{2}$ and $B=\frac{2 p-(N(p-2)+\alpha)}{2}$.

Proof. Taking $|f(x)|=|u(x)|^{p}, \quad|h(y)|=|u(y)|^{p}, \quad q=r=\frac{2 N}{2 N-\alpha}, \quad$ and applying Lemma 2.2, we get that

$$
\int_{\mathbb{R}^{N} \times \mathbb{R}^{N}} \frac{|u(x)|^{p}|u(y)|^{p}}{|x-y|^{\alpha}} d x d y \leq C\left(\int|u|^{\frac{2 N p}{2 N-\alpha}}\right)^{\frac{2 N-\alpha}{N}} .
$$

By the assumption of $(2 N-\alpha) / N<p<2^{*}(\alpha)$ and Lemma 2.1, we obtain that

$$
\int|u|^{\frac{2 N p}{2 N-\alpha}} \leq C\left(\int|\nabla u|^{2}\right)^{\frac{N(N p-2 N+\alpha)}{2(2 N-\alpha)}}\left(\int|u|^{2}\right)^{\frac{2 N p-N(N p-2 N+\alpha)}{2(2 N-\alpha)}} .
$$

It is now deduced from (2.2) and (2.3) that (2.1) holds.

Lemma 2.4. Let $N \geq 2,0<\alpha<N$ and $(2 N-\alpha) / N<p<2^{*}(\alpha)$. If $u_{n} \rightarrow u$ weakly in $H_{\text {radial }}^{1}\left(\mathbb{R}^{N}\right):=\left\{u \in H^{1}\left(\mathbb{R}^{N}\right) ; u(x)=u(|x|)\right\}$, then

$$
\int_{\mathbb{R}^{N} \times \mathbb{R}^{N}} \frac{\left|u_{n}(x)\right|^{p}\left|u_{n}(y)\right|^{p}}{|x-y|^{\alpha}} d x d y \rightarrow \int_{\mathbb{R}^{N} \times \mathbb{R}^{N}} \frac{|u(x)|^{p}|u(y)|^{p}}{|x-y|^{\alpha}} d x d y .
$$

Proof. Firstly, for positive numbers $a, b, c$, and $d$, there holds

$$
\begin{aligned}
2\left|a^{p} b^{p}-c^{p} d^{p}\right| & =\left|\left(b^{p}+d^{p}\right)\left(a^{p}-c^{p}\right)+\left(a^{p}+c^{p}\right)\left(b^{p}-d^{p}\right)\right| \\
& \leq\left(b^{p}+d^{p}\right)\left(a^{p-1}+c^{p-1}\right)|a-c|+\left(a^{p}+c^{p}\right)\left(b^{p-1}+d^{p-1}\right)|b-d| .
\end{aligned}
$$

Putting $a=\left|u_{n}(x)\right|, b=\left|u_{n}(y)\right|, c=|u(x)|, d=|u(y)|$, we obtain from Lemma 2.2 with $\beta=\alpha, r=q=\frac{2 N}{2 N-\alpha}$, and the fact that $\left\{u_{n}\right\}$ is bounded in $H_{\text {radial }}^{1}\left(\mathbb{R}^{N}\right)$ that

$$
\begin{aligned}
& \int_{\mathbb{R}^{N} \times \mathbb{R}^{N}} \frac{\left(\left|u_{n}(y)\right|^{p}+|u(y)|^{p}\right)\left(\left|u_{n}(x)\right|^{p-1}+|u(x)|^{p-1}\right)|| u_{n}(x)|-| u(x)||}{|x-y|^{\alpha}} d x d y \\
& \leq C_{1}\left[\int\left(\left.\left|u_{n}(x)-u(x)\right||| u_{n}(x)\right|^{p-1}+|u(x)|^{p-1} \mid\right)^{\frac{2 N}{2 N-\alpha}}\right]^{\frac{2 N-\alpha}{2 N}} \\
& \quad \times\left(\int\left(\left|u_{n}(y)\right|^{p}+|u(y)|^{p}\right)^{\frac{2 N}{2 N-\alpha}}\right)^{\frac{2 N-\alpha}{2 N}} \\
& \leq C_{2}\left[\left(\int\left|u_{n}(x)-u(x)\right|^{\frac{2 N p}{2 N-\alpha}}\right)^{\frac{1}{p}}\left(\int\left[\left|u_{n}(x)\right|^{\frac{2 N p}{2 N-\alpha}}+|u(x)|^{\frac{2 N p}{2 N-\alpha}}\right]\right)^{\frac{p}{p-1}}\right]^{\frac{2 N-\alpha}{2 N}} \\
& \quad \times\left[\int\left(\left|u_{n}(y)\right|^{\frac{2 N p}{2 N-\alpha}}+|u(y)|^{\frac{2 N p}{2 N-\alpha}}\right)\right]^{\frac{2 N-\alpha}{2 N}} \\
& \leq C_{3}\left(\int\left|u_{n}(x)-u(x)\right|^{\frac{2 N p}{2 N-\alpha}}\right)^{\frac{2 N-\alpha}{2 N p}} .
\end{aligned}
$$

It is now deduced from the Strauss lemma, i.e.,

$u_{n} \rightarrow u \quad$ weakly in $H_{\text {radial }}^{1}\left(\mathbb{R}^{N}\right)$ implies that $u_{n} \rightarrow u$ strongly in $L^{\frac{2 N p}{2 N-\alpha}}\left(\mathbb{R}^{N}\right)$,

that

$$
\int\left|u_{n}(x)-u(x)\right|^{\frac{2 N p}{2 N-\alpha}} \rightarrow 0 \quad \text { as } \quad n \rightarrow \infty .
$$


Therefore as $n \rightarrow \infty$, we have that

$$
\int_{\mathbb{R}^{N} \times \mathbb{R}^{N}} \frac{\left(\left|u_{n}(y)\right|^{p}+|u(y)|^{p}\right)\left(\left|u_{n}(x)\right|^{p-1}+|u(x)|^{p-1}\right)|| u_{n}(x)|-| u(x)||}{|x-y|^{\alpha}} d x d y \rightarrow 0 .
$$

By a similar argument, we obtain that as $n \rightarrow \infty$,

$$
\int_{\mathbb{R}^{N} \times \mathbb{R}^{N}} \frac{\left(\left|u_{n}(x)\right|^{p}+|u(x)|^{p}\right)\left(\left|u_{n}(y)\right|^{p-1}+|u(y)|^{p-1}\right)|| u_{n}(y)|-| u(y)||}{|x-y|^{\alpha}} d x d y \rightarrow 0 .
$$

Combining these with (IE), we easily obtain that

$$
\int_{\mathbb{R}^{N} \times \mathbb{R}^{N}} \frac{\left|u_{n}(x)\right|^{p}\left|u_{n}(y)\right|^{p}}{|x-y|^{\alpha}} d x d y \rightarrow \int_{\mathbb{R}^{N} \times \mathbb{R}^{N}} \frac{|u(x)|^{p}|u(y)|^{p}}{|x-y|^{\alpha}} d x d y .
$$

The proof is complete.

Now we are going to determine the best (smallest) positive constant $C(p, \alpha, N)$ such that (2.1) holds. This will be done by minimizing the functional

$$
J(u)=\frac{\left(\int|\nabla u|^{2}\right)^{A}\left(\int|u|^{2}\right)^{B}}{\int_{\mathbb{R}^{N} \times \mathbb{R}^{N}} \frac{|u(x)|^{p}|u(y)|^{p}}{|x-y|^{\alpha}} d x d y}, \quad u \in H^{1}\left(\mathbb{R}^{N}\right),
$$

where

$$
A=\frac{N(p-2)+\alpha}{2}, \quad B=\frac{2 p-(N(p-2)+\alpha)}{2} .
$$

According to inequality (2.1), the functional $J$ is well defined and $C^{1}$ in $H^{1}\left(\mathbb{R}^{N}\right)$. Moreover, we have that:

Lemma 2.5. Let $N \geq 2,0<\alpha<N,(2 N-\alpha) / N<p<(2 N-\alpha) /(N-2)$ when $N \geq 3$, and $(2 N-\alpha) / N<p<+\infty$ when $N=2$. Then

$$
m=\inf \left\{J(u) ; u \in H^{1}\left(\mathbb{R}^{N}\right), u \neq 0\right\}
$$

is attained by a function $\psi \in H^{1}\left(\mathbb{R}^{N}\right)$. Moreover $\psi$ is the minimal action solution of

$$
-2 A \triangle \psi+2 B \psi-2 m p \psi|\psi|^{p-2} \int_{\mathbb{R}^{N}} \frac{|\psi(y)|^{p}}{|x-y|^{\alpha}} d y=0, \quad \psi>0 \text { and } \psi \in H^{1}\left(\mathbb{R}^{N}\right)
$$

and

$$
m^{-1}=\int_{\mathbb{R}^{N} \times \mathbb{R}^{N}} \frac{|\psi(x)|^{p}|\psi(y)|^{p}}{|x-y|^{\alpha}} d x d y
$$

Proof. First, it is easy to see that for any $0 \neq u \in H^{1}\left(\mathbb{R}^{N}\right), J(u)>0$. (2.1) implies that we can find a sequence $\left\{v_{n}\right\} \subset H^{1}\left(\mathbb{R}^{N}\right)$ such that

$$
0<m=\lim _{n \rightarrow \infty} J\left(v_{n}\right)<+\infty .
$$

For any $\lambda, \mu>0$, if we set $u^{\lambda, \mu}(x)=\lambda u(\mu x)$ then we have that

$$
\int\left|\nabla u^{\lambda, \mu}(x)\right|^{2}=\lambda^{2} \mu^{2-N} \int|\nabla u|^{2},
$$




$$
\begin{gathered}
\int\left|u^{\lambda, \mu}(x)\right|^{2}=\lambda^{2} \mu^{-N} \int|u|^{2} \text { and } \\
\int_{\mathbb{R}^{N} \times \mathbb{R}^{N}} \frac{\left|u^{\lambda, \mu}(x)\right|^{p}\left|u^{\lambda, \mu}(y)\right|^{p}}{|x-y|^{\alpha}} d x d y=\lambda^{2 p} \mu^{\alpha-2 N} \int_{\mathbb{R}^{N} \times \mathbb{R}^{N}} \frac{|u(x)|^{p}|u(y)|^{p}}{|x-y|^{\alpha}} d x d y .
\end{gathered}
$$

It follows from the choice of $A$ and $B$ that

$$
J\left(u^{\lambda, \mu}\right)=\frac{\left(\lambda^{2} \mu^{2-N} \int|\nabla u|^{2}\right)^{A}\left(\lambda^{2} \mu^{-N} \int|u|^{2}\right)^{B}}{\lambda^{2 p} \mu^{\alpha-2 N} \int_{\mathbb{R}^{N} \times \mathbb{R}^{N}} \frac{|u(x)|^{p}|u(y)|^{p}}{|x-y|^{\alpha}} d x d y}=J(u) .
$$

Now let $\left\{u_{n}\right\} \subset H^{1}\left(\mathbb{R}^{N}\right)$ be a minimizing sequence of $m$. We can assume that $u_{n}>0$ since $\int|\nabla| u_{n}||^{2} \leq \int\left|\nabla u_{n}\right|^{2}$. Furthermore, by symmetrization [3, 19, 4], we can take $u_{n}(x)=u_{n}(|x|)$. Choosing

$$
\mu_{n}=\frac{\left\|u_{n}\right\|_{2}}{\left\|\nabla u_{n}\right\|_{2}}, \quad \lambda_{n}=\frac{\left\|u_{n}\right\|_{2}^{\frac{N}{2}}-1}{\left\|\nabla u_{n}\right\|_{2}^{\frac{N}{2}}},
$$

we obtain a sequence $\psi_{n}(x)=u_{n}^{\lambda_{n}, \mu_{n}}(x)$ satisfying the following properties:

$$
\begin{gathered}
\psi_{n} \in H^{1}\left(\mathbb{R}^{N}\right), \quad \psi_{n}(x)=\psi_{n}(|x|) \quad \text { and } \quad \psi_{n} \geq 0 \\
\left\|\psi_{n}\right\|_{2}^{2}=1 \quad \text { and } \quad\left\|\nabla \psi_{n}\right\|_{2}^{2}=1 \\
m=\lim _{n \rightarrow \infty} J\left(\psi_{n}\right) .
\end{gathered}
$$

Going if necessary to a subsequence, still denoted by $\left\{\psi_{n}\right\}$, we may assume that $\psi_{n} \rightarrow \psi$ weakly in $H_{\text {radial }}^{1}\left(\mathbb{R}^{N}\right)$. It is now deduced from Lemma 2.4 that

$$
\int_{\mathbb{R}^{N} \times \mathbb{R}^{N}} \frac{\left|\psi_{n}(x)\right|^{p}\left|\psi_{n}(y)\right|^{p}}{|x-y|^{\alpha}} d x d y \rightarrow \int_{\mathbb{R}^{N} \times \mathbb{R}^{N}} \frac{|\psi(x)|^{p}|\psi(y)|^{p}}{|x-y|^{\alpha}} d x d y .
$$

On the other hand, by weak convergence $\|\psi\|_{2} \leq 1$ and $\|\nabla \psi\|_{2} \leq 1$. Therefore

$$
m \leq J(\psi) \leq \frac{1}{\int_{\mathbb{R}^{N} \times \mathbb{R}^{N}} \frac{\left.|\psi(x)|\right|^{p}|\psi(y)|^{p}}{|x-y|^{\alpha}} d x d y}=\lim _{n \rightarrow \infty} J\left(\psi_{n}\right)=m .
$$

It follows that $\left(\int|\nabla \psi|^{2}\right)^{A}\left(\int|\psi|^{2}\right)^{B}=1$ and hence $\|\psi\|_{2}=1$ and $\|\nabla \psi\|_{2}=1$. So $\psi_{n} \rightarrow \psi$ strongly in $H^{1}\left(\mathbb{R}^{N}\right)$ and

$$
m=J(\psi)=\frac{\left(\int|\nabla \psi|^{2}\right)^{\frac{A}{2}}\left(\int|\psi|^{2}\right)^{\frac{B}{2}}}{\int_{\mathbb{R}^{N} \times \mathbb{R}^{N}} \frac{|\psi(x)|^{p}|\psi(y)|^{p}}{|x-y|^{\alpha}} d x d y}=\left(\int_{\mathbb{R}^{N} \times \mathbb{R}^{N}} \frac{|\psi(x)|^{p}|\psi(y)|^{p}}{|x-y|^{\alpha}} d x d y\right)^{-1} .
$$

Noticing that

$$
\left.\frac{d}{d \varepsilon}\right|_{\varepsilon=0} J(\psi+\varepsilon \eta)=0 \quad \text { for all } \quad \eta \in C_{0}^{\infty}\left(\mathbb{R}^{N}\right),
$$

we have from a direct computation that $\psi$ satisfies (2.4). 
REMARK 2.6. Here and after, a minimal action solution of an equation is defined in the following sense. For example, for equation

$$
-\triangle u+u-u|u|^{p-2} \int_{\mathbb{R}^{N}} \frac{|u(y)|^{p}}{|x-y|^{\alpha}} d y=0, \quad u \in H^{1}\left(\mathbb{R}^{N}\right)
$$

and functional

$$
L(u)=\int \frac{|\nabla u|^{2}+|u|^{2}}{2}-\frac{1}{2 p} \int_{\mathbb{R}^{N} \times \mathbb{R}^{N}} \frac{|u(x)|^{p}|u(y)|^{p}}{|x-y|^{\alpha}} d x d y, \quad u \in H^{1}\left(\mathbb{R}^{N}\right),
$$

we give the following definitions. The set of solutions of equation (2.5) is denoted by $\Gamma$, namely

$$
\Gamma=\left\{\phi \in H^{1}\left(\mathbb{R}^{N}\right) ; \quad \phi \neq 0, \quad L^{\prime}(\phi)=0\right\}
$$

The set of minimal action solutions of equation (2.5) is denoted by $G$, namely

$$
G=\{\phi \in \Gamma ; \quad L(\phi) \leq L(\psi) \text { for any } \psi \in \Gamma\}
$$

TheOREM 2.7. Suppose the assumptions of Lemma 2.5 hold. The best constant $C(p, \alpha, N)$ in inequality (2.1) is exactly given as

$$
\begin{aligned}
C(p, \alpha, N) & =\frac{p}{B}\left(\frac{B}{A}\right)^{A}\|w\|_{2}^{2-2 p} \\
& =\frac{2 p}{2 p-(N(p-2)+\alpha)}\left(\frac{2 p-(N(p-2)+\alpha)}{N(p-2)+\alpha}\right)^{\frac{N(p-2)+\alpha}{2}}\|w\|_{2}^{2-2 p},
\end{aligned}
$$

where $w$ is the minimal action solution of

$$
-\triangle \phi+\phi-\phi|\phi|^{p-2} \int_{\mathbb{R}^{N}} \frac{|\phi(y)|^{p}}{|x-y|^{\alpha}} d y=0, \quad \phi>0 \text { and } \phi \in H^{1}\left(\mathbb{R}^{N}\right) .
$$

REMARK 2.8. We emphasize that when $p=2+\frac{2-\alpha}{N}$ and $w$ is a minimal action solution of (2.6), then the following

$$
\varphi(x, t)=(T-t)^{-\frac{N}{2}} e^{-\frac{i|x|^{2}}{4(T-t)}} w\left(\frac{x}{T-t}\right) e^{\frac{i t}{T(T-t)}}
$$

is a solution of $i \varphi_{t}+\triangle \varphi+\varphi|\varphi|^{p-2}\left(|x|^{-\alpha} *|\varphi|^{p}\right)=0$ and $\varphi$ blows up at finite time. But for general $p$, the blow-up derived by self-similarity is still open and we can not solve it at this moment. Actually, considering the Schrodinger equation with local nonlinearity $i \varphi_{t}=-\Delta \varphi-|\varphi|^{q} \varphi$, one can get the blowup profile by self-similarity and pseudo-conformal invariance holds only for $q=\frac{4}{N}$. These are problems for further study.

\section{Proof of Theorem 2.7.}

Proof. Scaling

$$
\psi(x)=(2 p m)^{-\frac{1}{2 p-2}} u(x)
$$


we know from Lemma 2.5 that $u(x)$ satisfies

$$
-2 A \triangle \phi+2 B \phi-\phi|\phi|^{p-2} \int_{\mathbb{R}^{N}} \frac{|\phi(y)|^{p}}{|x-y|^{\alpha}} d y=0, \quad \phi>0 \text { and } \phi \in H^{1}\left(\mathbb{R}^{N}\right) .
$$

A second scaling

$$
u(x)=\left(2 B\left(\frac{B}{A}\right)^{\frac{N-\alpha}{2}}\right)^{\frac{1}{2 p-2}} w\left(\left(\frac{B}{A}\right)^{\frac{1}{2}} x\right)
$$

shows that $w(x)$ is the ground state solution of equation (2.6). Now using

$$
\psi(x)=(2 p m)^{-\frac{1}{2 p-2}}\left(2 B\left(\frac{B}{A}\right)^{\frac{N-\alpha}{2}}\right)^{\frac{1}{2 p-2}} w\left(\left(\frac{B}{A}\right)^{\frac{1}{2}} x\right)
$$

and the fact that $\|\psi\|_{2}=1$, we obtain that

$$
\begin{aligned}
C(p, \alpha, N) & =m^{-1}=\frac{p}{B}\left(\frac{B}{A}\right)^{A}\|w\|_{2}^{2-2 p} \\
& =\frac{2 p}{2 p-(N(p-2)+\alpha)}\left(\frac{2 p-(N(p-2)+\alpha)}{N(p-2)+\alpha}\right)^{\frac{N(p-2)+\alpha}{2}}\|w\|_{2}^{2-2 p} .
\end{aligned}
$$

The proof is complete.

REMARK 2.9. We emphasize that although we do not know whether the minimal action solution of equation (2.6) is unique or not, the best constant $C(p, \alpha, N)$ is independent of the choice of $w$. Indeed, denote $d=\inf \{L(u) ; u \in \Gamma\}$, then for a minimal action solution $w$ of equation (2.6), we have that $0<d=L(w)<+\infty$ and $d$ is independent of the choice of $w$; see e.g. [9]. On the other hand, since $w$ is a solution of equation (2.6) we have that

$$
\begin{gathered}
\int|\nabla w|^{2}-\frac{N p+\alpha-2 N}{2 p} \int_{\mathbb{R}^{N} \times \mathbb{R}^{N}} \frac{|w(x)|^{p}|w(y)|^{p}}{|x-y|^{\alpha}} d x d y=0 \text { and } \\
\int\left(|\nabla w|^{2}+|w|^{2}\right)-\frac{N p+\alpha-2 N}{2 p} \int_{\mathbb{R}^{N} \times \mathbb{R}^{N}} \frac{|w(x)|^{p}|w(y)|^{p}}{|x-y|^{\alpha}} d x d y=0 .
\end{gathered}
$$

Therefore

$$
\int_{\mathbb{R}^{N} \times \mathbb{R}^{N}} \frac{|w(x)|^{p}|w(y)|^{p}}{|x-y|^{\alpha}} d x d y=\frac{2 p}{2 p-(N(p-2)+\alpha)} \int|w|^{2}
$$

and

$$
\int|\nabla w|^{2}=\frac{N(p-2)+\alpha}{2 p-(N(p-2)+\alpha)} \int|w|^{2} .
$$

It is deduced that

$$
d=L(w)=\frac{p-1}{2 p-(N(p-2)+\alpha)}\|w\|_{L^{2}}^{2},
$$

which is equivalent to

$$
\|w\|_{L^{2}}=\left(\frac{2 p-(N(p-2)+\alpha)}{p-1} d\right)^{\frac{1}{2}} .
$$

Thus $C(p, \alpha, N)$ is independent of the choice of $w$. 


\section{Cauchy problem}

In this section, we sketch some results on the existence of a local or global solution of equations (1.2)-(1.3). Define

$$
\Sigma=\left\{u \in H^{1}\left(\mathbb{R}^{N}\right) ; \int|x|^{2}|u|^{2}<+\infty\right\} .
$$

Then $\Sigma$ is a Hilbert space under the inner product

$$
\langle u, v\rangle_{\Sigma}=\operatorname{Re} \int\left(\nabla u \nabla \bar{v}+|x|^{2} u \bar{v}+u \bar{v}\right) .
$$

The norm on $\Sigma$ is denoted by $\|u\|_{\Sigma}^{2}=\int\left(|\nabla u|^{2}+|x|^{2}|u|^{2}+|u|^{2}\right)$. The following Proposition is proved in the appendix for the readers convenience.

Proposition 3.1. Let $0<\alpha<\min \{N, 4\}$ and $2 \leq p<2^{*}(\alpha)$. For any $\varphi_{0} \in \Sigma$, there is a $T=T\left(\left\|\varphi_{0}\right\|_{\Sigma}\right)>0$ and a unique solution $\varphi$ of equation (1.2) with $\varphi \in C([0, T), \Sigma)$ and $\varphi(0)=\varphi_{0}$. Moreover, we have conservation of particle number

$$
\int|\varphi|^{2} \equiv \int\left|\varphi_{0}\right|^{2}
$$

and conservation of energy

$$
E(\varphi)=\frac{1}{2} \int\left(|\nabla \varphi|^{2}+|x|^{2}|\varphi|^{2}\right)-\frac{1}{2 p} \int_{\mathbb{R}^{N} \times \mathbb{R}^{N}} \frac{|\varphi(x)|^{p}|\varphi(y)|^{p}}{|x-y|^{\alpha}} d x d y=E\left(\varphi_{0}\right)
$$

for all $t \in[0, T)$, where either $T=+\infty$ or $T<+\infty$ and $\lim _{t \rightarrow T^{-}}\|\varphi\|_{\Sigma}=+\infty$.

Theorem 3.2. Let $0<\alpha<\min \{N, 4\}$ and $2 \leq p<2^{*}(\alpha)$.

- If $2 \leq p<2+(2-\alpha) / N$, then for any $\varphi_{0} \in \Sigma$ the solution $\varphi(x, t)$ of equation (1.2) exists globally in time.

- If $2 \leq p=2+(2-\alpha) / N$, then the solution $\varphi(x, t)$ of equation (1.2) exists globally in time provided the initial data $\left\|\varphi_{0}\right\|_{L^{2}}$ sufficiently small.

Proof. Let $\varphi(x, t) \in C([0, T), \Sigma)$ be the solution of equation (1.2) with initial data $\varphi_{0}$. By Proposition 3.1 and Theorem 2.3, we have that

$$
\begin{aligned}
E\left(\varphi_{0}\right)= & E(\varphi) \\
\geq & \frac{1}{2} \int\left(|\nabla \varphi|^{2}+|x|^{2}|\varphi|^{2}\right)-C\left(\int|\nabla \varphi|^{2}\right)^{\frac{N(p-2)+\alpha}{2}}\left(\int|\varphi|^{2}\right)^{\frac{2 p-(N(p-2)+\alpha)}{2}} \\
\geq & \frac{1}{2}\left(|\nabla \varphi|^{2}+|x|^{2}|\varphi|^{2}\right) \\
& \quad-C\left(\int\left(|\nabla \varphi|^{2}+|x|^{2}|\varphi|^{2}\right)\right)^{\frac{N(p-2)+\alpha}{2}}\left(\int|\varphi|^{2}\right)^{\frac{2 p-(N(p-2)+\alpha)}{2}} .
\end{aligned}
$$

If $2 \leq p<2+(2-\alpha) / N$, then the Young inequality implies that there is $0<\varepsilon<\frac{1}{2}$ and $C_{\varepsilon}$ such that

$$
E\left(\varphi_{0}\right) \geq\left(\frac{1}{2}-\varepsilon\right) \int\left(|\nabla \varphi|^{2}+|x|^{2}|\varphi|^{2}\right)-C_{\varepsilon}\left(\int|\varphi|^{2}\right)^{\frac{2 p-(N(p-2)+\alpha)}{2-(N(p-2)+\alpha)}} .
$$

Hence $\int\left(|\nabla \varphi|^{2}+|x|^{2}|\varphi|^{2}\right)$ is bounded with respect to $t$. Proposition 3.1 implies that $\varphi(x, t)$ exists globally in time. 
If $p=2+(2-\alpha) / N$, then

$$
\begin{aligned}
E\left(\varphi_{0}\right) & \geq \frac{1}{2} \int\left(|\nabla \varphi|^{2}+|x|^{2}|\varphi|^{2}\right)-C \int|\nabla \varphi|^{2}\left(\int|\varphi|^{2}\right)^{p-1} \\
& \geq \frac{1}{2} \int\left(|\nabla \varphi|^{2}+|x|^{2}|\varphi|^{2}\right)-C \int\left(|\nabla \varphi|^{2}+|x|^{2}|\varphi|^{2}\right)\left(\int|\varphi|^{2}\right)^{p-1} \\
& \geq C_{1} \int\left(|\nabla \varphi|^{2}+|x|^{2}\left|\varphi_{0}\right|^{2}\right)
\end{aligned}
$$

provided $\left\|\varphi_{0}\right\|_{L^{2}}$ sufficiently small. It follows that the solution $\varphi(x, t)$ of equation (1.2) exists globally in time when $p=2+(2-\alpha) / N$ and $\left\|\varphi_{0}\right\|_{L^{2}}$ is small enough.

REMARK 3.3. It is observed that in Theorem 3.2, " $\left\|\varphi_{0}\right\|_{L^{2}}$ small enough" is vague. It is naturally to ask: how small? This is one of the goals of the next section.

\section{Critical mass for critical nonlinearity}

In this section, we will use "our best constant" to give a sharp condition on the solution of equations (1.2)-(1.3) which exists globally in time or blows up in a finite time. In particular, we give an answer to the question: how small an initial data can ensure the existence of global solution of equations (1.2)-(1.3) in the case of $p=2+(2-\alpha) / N ?$ The answer is simple, as we see below.

Theorem 4.1. Let $N \geq 2,0<\alpha<\min \{N, 4\}$, and $p=2+(2-\alpha) / N$. If $\varphi_{0} \in \Sigma$ and

$$
\left\|\varphi_{0}\right\|_{L^{2}}<\|w\|_{L^{2}}
$$

where $w$ is a minimal action solution of equations (2.6), then equation (1.2)-(1.3) has a global solution $\varphi(x, t) \in C\left(\mathbb{R}_{+}, \Sigma\right)$.

Proof. Let $\varphi(x, t) \in C([0, T), \Sigma)$ be a solution of equations (1.2)-(1.3) in the case of $p=2+(2-\alpha) / N$. Using Theorem 2.7, one has

$$
\int_{\mathbb{R}^{N} \times \mathbb{R}^{N}} \frac{|\varphi(x)|^{p}|\varphi(y)|^{p}}{|x-y|^{\alpha}} d x d y \leq \frac{2 N+2-\alpha}{N}\left(\frac{\int|\varphi|^{2}}{\int|w|^{2}}\right)^{\frac{N+2-\alpha}{N}} \int|\nabla \varphi|^{2} .
$$

Combining (4.2) with the conservation of energy equation (3.2), we obtain that

$$
\begin{aligned}
E\left(\varphi_{0}\right) & =\frac{1}{2} \int\left(|\nabla \varphi|^{2}+|x|^{2}|\varphi|^{2}\right)-\frac{N}{2(2 N+2-\alpha)} \int_{\mathbb{R}^{N} \times \mathbb{R}^{N}} \frac{|\varphi(x)|^{p}|\varphi(y)|^{p}}{|x-y|^{\alpha}} d x d y \\
& \geq \frac{1}{2}\left[1-\left(\frac{\int|\varphi|^{2}}{\int|w|^{2}}\right)^{\frac{N+2-\alpha}{N}}\right] \int|\nabla \varphi|^{2}+\frac{1}{2} \int|x|^{2}|\varphi|^{2} .
\end{aligned}
$$

As

$$
\int|\varphi|^{2} \equiv \int\left|\varphi_{0}\right|^{2}<\int|w|^{2}
$$

both $\int|\nabla \varphi|^{2}$ and $\int|x|^{2}|\varphi|^{2}$ are bounded for $t \in[0, T)$. It is deduced from Proposition 3.1 that $\varphi(x, t)$ exists globally in $t \in[0,+\infty)$.

REMARK 4.2. We point out that we are studying the Schrodinger equation with harmonic potential, and the condition (4.1) is sharp in the sense of the following theorem. 
Theorem 4.3. Let $N \geq 2,0<\alpha<\min \{N, 4\}$, and $p=2+(2-\alpha) / N$. If $\varphi_{0} \in \Sigma$ with the form

$$
\varphi_{0}(x)=c \lambda^{\frac{N}{2}} w(\lambda x),
$$

where $\lambda>0, w$ is the minimal action solution of (2.6), and $c$ is a complex number with $|c| \geq 1$, then

$$
\left\|\varphi_{0}\right\|_{L^{2}} \geq\|w\|_{L^{2}}
$$

Moreover the solution $\varphi(x, t)$ of equations (1.2)-(1.3) must blow up in a finite time.

In order to prove Theorem 4.3, we need several lemmas. Firstly, we have the following virial identity which originated from Glassey [16].

Proposition 4.4. Let $N \geq 2,0<\alpha<\min \{N, 4\}$. Let $\varphi_{0} \in \Sigma$ and $\varphi \in C([0, T), \Sigma)$ be the solution of equations (1.2)-(1.3). If $h(t)=\frac{1}{2} \int|x|^{2}|\varphi|^{2}$ and $p=2+(2-\alpha) / N$, then one has

$$
h^{\prime \prime}(t)=8 E\left(\varphi_{0}\right)-16 h(t) .
$$

Proof. We only prove equation (4.5) formally. Since $\varphi$ satisfies equation (1.2), we have that

$$
\varphi_{t}=i\left(\triangle \varphi-|x|^{2} \varphi+\varphi|\varphi|^{p-2} \int_{\mathbb{R}^{N}} \frac{|\varphi(y)|^{p}}{|x-y|^{\alpha}} d y\right)
$$

Therefore

$$
h^{\prime}(t)=\operatorname{Re} \int|x|^{2} \bar{\varphi} \varphi_{t}=2 \operatorname{Im} \int \bar{\varphi} x \nabla \varphi
$$

and

$$
\begin{aligned}
h^{\prime \prime}(t) & =2 \operatorname{Im} \int\left(\bar{\varphi}_{t} x \nabla \varphi+\bar{\varphi} x \nabla \varphi_{t}\right) \\
& =2 \operatorname{Im} \int \bar{\varphi}_{t} x \nabla \varphi-2 \operatorname{Im} \int \varphi_{t}(N \bar{\varphi}+x \nabla \bar{\varphi}) \\
& =-2 \operatorname{Im} \int \varphi_{t}(N \bar{\varphi}+2 x \nabla \bar{\varphi}) \\
& =-2 \operatorname{Re} \int(N \bar{\varphi}+2 x \nabla \bar{\varphi})\left(\triangle \varphi-|x|^{2} \varphi+\varphi|\varphi|^{p-2} \int_{\mathbb{R}^{N}} \frac{|\varphi(y)|^{p}}{|x-y|^{\alpha}} d y\right) .
\end{aligned}
$$

Direct computations show that

$$
\begin{gathered}
\operatorname{Re} \int(N \bar{\varphi}+2 x \nabla \bar{\varphi}) \triangle \varphi=-2 \int|\nabla \varphi|^{2} ; \\
\operatorname{Re} \int(N \bar{\varphi}+2 x \nabla \bar{\varphi})|x|^{2} \varphi=-2 \int|x|^{2}|\varphi|^{2} ; \\
\operatorname{Re} \int(N \bar{\varphi}+2 x \nabla \bar{\varphi}) \varphi|\varphi|^{p-2} \int_{\mathbb{R}^{N}} \frac{|\varphi(y)|^{p}}{|x-y|^{\alpha}} d y \\
=N \int_{\mathbb{R}^{N} \times \mathbb{R}^{N}} \frac{|\varphi(x)|^{p}|\varphi(y)|^{p}}{|x-y|^{\alpha}} d x d y+\operatorname{Re} \int 2 x \nabla \bar{\varphi} \varphi|\varphi|^{p-2} \int_{\mathbb{R}^{N}} \frac{|\varphi(y)|^{p}}{|x-y|^{\alpha}} d y .
\end{gathered}
$$


Since

$$
\operatorname{Re} \int 2 x \nabla \bar{\varphi} \varphi|\varphi|^{p-2} \int_{\mathbb{R}^{N}} \frac{|\varphi(y)|^{p}}{|x-y|^{\alpha}} d y=\frac{\alpha-2 N}{p} \int_{\mathbb{R}^{N} \times \mathbb{R}^{N}} \frac{|\varphi(x)|^{p}|\varphi(y)|^{p}}{|x-y|^{\alpha}} d x d y,
$$

we obtain that

$\operatorname{Re} \int(N \bar{\varphi}+2 x \nabla \bar{\varphi}) \varphi|\varphi|^{p-2} \int_{\mathbb{R}^{N}} \frac{|\varphi(y)|^{p}}{|x-y|^{\alpha}} d y=\frac{N(p-2)+\alpha}{p} \int_{\mathbb{R}^{N} \times \mathbb{R}^{N}} \frac{|\varphi(x)|^{p}|\varphi(y)|^{p}}{|x-y|^{\alpha}} d x d y$.

It is now deduced that

$$
\begin{aligned}
h^{\prime \prime}(t) & =4\left(\int\left(|\nabla \varphi|^{2}-|x|^{2}|\varphi|^{2}\right)-\frac{N(p-2)+\alpha}{2 p} \int_{\mathbb{R}^{N} \times \mathbb{R}^{N}} \frac{|\varphi(x)|^{p}|\varphi(y)|^{p}}{|x-y|^{\alpha}} d x d y\right) \\
& =8 E(\varphi)-16 h(t) .
\end{aligned}
$$

Lemma 4.5. Let $N \geq 2,0<\alpha<\min \{N, 4\}$, and $p=2+(2-\alpha) / N$. If $\varphi_{0} \not \equiv 0$ satisfies that

$$
h(0)=\frac{1}{2} \int|x|^{2}\left|\varphi_{0}\right|^{2} \geq E\left(\varphi_{0}\right)
$$

then the solution $\varphi$ of equations (1.2)-(1.3) blows up in a finite time.

Proof. From Proposition 4.4, we have that

$$
h(t)=\beta \sin (4 t+\theta)+\frac{1}{2} E\left(\varphi_{0}\right)
$$

where $\beta$ and $\theta$ are constants determined by $h(0)$ and $h^{\prime}(0)$. Moreover,

$$
\beta^{2}=\left(h(0)-\frac{1}{2} E\left(\varphi_{0}\right)\right)^{2}+\frac{1}{16}\left(h^{\prime}(0)\right)^{2} .
$$

Thus if $h(0) \geq E\left(\varphi_{0}\right),(4.6)$ and (4.7) imply that there exists $T_{0}<\infty$ such that

$$
\lim _{t \rightarrow T_{0}^{-}} h(t)=0 .
$$

It is deduced from (see e.g. [24])

$$
\int|\varphi|^{2} \leq C\left(\int|x|^{2}|\varphi|^{2}\right)^{\frac{1}{2}}\left(\int|\nabla \varphi|^{2}\right)^{\frac{1}{2}}
$$

that there exists $0<T<+\infty$ such that

$$
\lim _{t \rightarrow T^{-}} \int|\nabla \varphi|^{2}=+\infty
$$

This proves that $\varphi(x, t)$ blows up in a finite time.

\section{Proof of Theorem 4.3.}

Proof. For any positive constant $\lambda$ and complex number $c$ with $|c| \geq 1$, a direct computation yields that

$$
\int\left|\varphi_{0}\right|^{2}=|c|^{2} \int\left|\lambda^{\frac{N}{2}} w(\lambda x)\right|^{2} d x=|c|^{2} \int|w|^{2} \geq \int|w|^{2}
$$


On the other hand, since the function $w(x)$ makes the inequality (2.1) into an equality, one has that

$$
\int_{\mathbb{R}^{N} \times \mathbb{R}^{N}} \frac{|w(x)|^{p}|w(y)|^{p}}{|x-y|^{\alpha}} d x d y=\frac{2 N+2-\alpha}{N} \int|\nabla w|^{2} .
$$

Therefore

$$
\begin{aligned}
E\left(\varphi_{0}\right) & =\frac{1}{2} \int\left|\nabla \varphi_{0}\right|^{2}-\frac{N}{2(2 N+2-\alpha)} \int_{\mathbb{R}^{N} \times \mathbb{R}^{N}} \frac{\left|\varphi_{0}(x)\right|^{p}\left|\varphi_{0}(y)\right|^{p}}{|x-y|^{\alpha}} d x d y+h(0) \\
& =\frac{1}{2}\left(1-|c|^{\frac{2 N+4-2 \alpha}{N}}\right) \lambda^{2}|c|^{2} \int|\nabla w|^{2}+h(0) \\
& \leq h(0) .
\end{aligned}
$$

It follows from Lemma 4.5 that $\varphi(x, t)$ blows up in a finite time. The proof of Theorem 4.3 is complete.

REMARK 4.6. From Theorem 4.1 and 4.3 , we see that when $p=2+(2-\alpha) / N,\|w\|_{L^{2}}$ is the critical mass for the solutions of equations (1.2)-(1.3) which exist globally in time. The prescribed initial data in Theorem 4.3 also implies that the existence of blow-up solutions of equations (1.2)-(1.3) not only depends on the mass of the initial data but also on the profile of the initial data. So it is very reasonable to conjecture that for some class of initial data $\varphi_{0}$ with $\left\|\varphi_{0}\right\|_{L^{2}} \geq\|w\|_{L^{2}}$, the solutions of equations (1.2)-(1.3) exist globally in time. In fact, this conjecture is true in the case of $2+(2-\alpha) / N<p<2^{*}(\alpha)$. Furthermore, we can prove that when $2+(2-\alpha) / N<$ $p<2^{*}(\alpha)$, the solutions of equations (1.2)-(1.3) exist globally in time for a large class of initial data whose norm can be taken as large as one wants.

\section{Global solutions for supercritical nonlinearity}

After developing the critical mass for the existence of global solutions and the blow-up solutions of equations (1.2)-(1.3) in the critical nonlinearity $p=2+(2-\alpha) / N$, attention is now focused on the existence of global solutions of equations (1.2)-(1.3) in the case of supercritical nonlinearity $2+(2-\alpha) / N<p<2^{*}(\alpha)$. An interesting aspect is that we can obtain global solutions for arbitrarily large data. We emphasize that the use of Theorem 2.7 is essential. First we need the following lemma from Bégout [2].

Lemma 5.1. Let $I \subset \mathbb{R}$ be an open interval, $s_{0} \in I, \theta>1, a>0, b>0$ and $\Phi(s) \in$ $C\left(I, \mathbb{R}_{+}\right)$. Set $f(y)=a-y+b y^{\theta}$ for any $y \geq 0$. Define $y_{*}=(b \theta)^{-\frac{1}{\theta-1}}$ and $b_{*}=\frac{\theta-1}{\theta} y_{*}$. Assume that $\Phi\left(s_{0}\right)<y_{*}, a \leq b_{*}$, and $f \circ \Phi>0$. Then $\Phi(s)<y_{*}$ for any $s \in I$.

Proof. Since $\Phi\left(s_{0}\right)<y_{*}$ and $\Phi$ is a continuous function, there exists a $\delta>0$ such that $\Phi(s)<y_{*}$ for any $s \in\left(s_{0}-\delta, s_{0}+\delta\right) \subset I$. If $\Phi(s)<y_{*}$ were not true for any $s \in I$, by continuity there would exist a $s_{*} \in I$ satisfying $\Phi\left(s_{*}\right)=y_{*}$. Then $f \circ \Phi\left(s_{*}\right)=f\left(y_{*}\right)=$ $a-b_{*} \leq 0$. However, this is impossible from $f \circ \Phi>0$. Therefore $\Phi(s)<y_{*}$ for any $s \in I$. The proof is complete.

Next, we define a real valued function $V(\lambda)$ as follows,

$$
V(\lambda)=\left(\frac{A-1}{B}\right)^{\frac{A-1}{2 B}}\|w\|_{L^{2}}^{\frac{p-1}{B}} \lambda^{-\frac{A-1}{2 B}}, \quad \lambda>0,
$$

where $A=\frac{N(p-2)+\alpha}{2}$ and $B=\frac{2 p-(N(p-2)+\alpha)}{2}$. Denote

$$
\mathcal{S}=\left\{u \in \Sigma ;\|u\|_{L^{2}} \leq V\left(\|\nabla u\|_{L^{2}}^{2}+\|x u\|_{L^{2}}^{2}\right)\right\} .
$$


Lemma 5.2. Let $N \geq 2,0<\alpha<\min \{N, 4\}$, and $2+(2-\alpha) / N<p<2^{*}(\alpha)$. $\mathcal{S}$ is an unbounded subset of $\Sigma$.

Proof. For any $M>0$, we choose $v \in \Sigma$ such that $\int|x|^{2}|v|^{2}>M$. Define $u_{\lambda}(x)=$ $\lambda^{\frac{N+2}{2}} v(\lambda x)(\lambda>0)$, then we have from direct computations that

$$
\begin{gathered}
\int|x|^{2}\left|u_{\lambda}(x)\right|^{2} d x=\int|x|^{2} \lambda^{N+2}|v(\lambda x)|^{2} d x=\int|x|^{2}|v|^{2} d x>M, \\
\int\left|\nabla u_{\lambda}(x)\right|^{2} d x=\lambda^{4} \int|\nabla v|^{2} d x \quad \text { and } \quad \int\left|u_{\lambda}(x)\right|^{2} d x=\lambda^{2} \int|v|^{2} d x .
\end{gathered}
$$

Therefore for $\lambda$ small enough, we have

$$
\begin{aligned}
\left\|u_{\lambda}\right\|_{L^{2}}\left(\left\|\nabla u_{\lambda}\right\|_{L^{2}}^{2}+\left\|x u_{\lambda}\right\|_{L^{2}}^{2}\right)^{\frac{A-1}{2 B}} & =\lambda\|v\|_{L^{2}}\left(\lambda^{4}\|\nabla v\|_{L^{2}}^{2}+\|x v\|_{L^{2}}^{2}\right)^{\frac{A-1}{2 B}} \\
& <\left(\frac{A-1}{B}\right)^{\frac{A-1}{2 B}}\|w\|_{L^{2}}^{\frac{p-1}{B}} .
\end{aligned}
$$

According to the definition of $\mathcal{S}$, we obtain that $u_{\lambda} \in \mathcal{S}$ for $\lambda$ small enough. On the other hand,

$$
\left\|u_{\lambda}\right\|_{\Sigma}^{2}>\int|x|^{2}\left|u_{\lambda}\right|^{2}=\int|x|^{2}|v|^{2} d x>M
$$

These prove that $\mathcal{S}$ is unbounded in $\Sigma$.

Theorem 5.3. Let $N \geq 2,0<\alpha<\min \{N, 4\}$ and $2+(2-\alpha) / N<p<2^{*}(\alpha)$. If $\varphi_{0} \in \mathcal{S}$, then the solutions $\varphi(x, t)$ of equations (1.2)-(1.3) exist globally in $t \in[0,+\infty)$. Moreover for any $t \in(0, T)$ we have that

$$
\left\|\varphi_{0}\right\|_{L^{2}}^{\frac{2 B}{A-1}}\left(\|\nabla \varphi(t)\|_{L^{2}}^{2}+\|x \varphi(t)\|_{L^{2}}^{2}\right)<\frac{A}{B}\|w\|_{L^{2}}^{\frac{2(p-1)}{A-1}}
$$

and

$$
\|\varphi(t)\|_{\Sigma}^{2} \leq \frac{2 N(p-2)+2 \alpha}{N(p-2)+\alpha-2} E\left(\varphi_{0}\right)+\left\|\varphi_{0}\right\|_{L^{2}}^{2}
$$

Proof. For any $t \in[0, T)$, applying Theorem 2.7 to $\varphi(t, x)$ and using the choice of $A$ and $B$, we obtain that

$$
\int_{\mathbb{R}^{N} \times \mathbb{R}^{N}} \frac{|\varphi(x)|^{p}|\varphi(y)|^{p}}{|x-y|^{\alpha}} d x d y \leq C(p, \alpha, N)\left\|\varphi_{0}\right\|_{L^{2}}^{2 B}\|\nabla \varphi\|_{L^{2}}^{2 A}
$$

Denote $a=\int\left(\left|\nabla \varphi_{0}\right|^{2}+|x|^{2}\left|\varphi_{0}\right|^{2}\right)>0$. It is deduced from the energy identity and (5.2) that

$$
\begin{aligned}
\int\left(|\nabla \varphi|^{2}+|x|^{2}|\varphi|^{2}\right) & =2 E(\varphi)+\frac{1}{p} \int_{\mathbb{R}^{N} \times \mathbb{R}^{N}} \frac{|\varphi(x)|^{p}|\varphi(y)|^{p}}{|x-y|^{\alpha}} d x d y \\
& =2 E\left(\varphi_{0}\right)+\frac{1}{p} \int_{\mathbb{R}^{N} \times \mathbb{R}^{N}} \frac{|\varphi(x)|^{p}|\varphi(y)|^{p}}{|x-y|^{\alpha}} d x d y \\
& <a+\frac{1}{p} \int_{\mathbb{R}^{N} \times \mathbb{R}^{N}} \frac{|\varphi(x)|^{p}|\varphi(y)|^{p}}{|x-y|^{\alpha}} d x d y \\
& \leq a+\frac{C(p, \alpha, N)}{p}\left\|\varphi_{0}\right\|_{L^{2}}^{2 B}\|\nabla \varphi\|_{L^{2}}^{2 A} \\
& \leq a+\frac{C(p, \alpha, N)}{p}\left\|\varphi_{0}\right\|_{L^{2}}^{2 B}\left(\int\left(|\nabla \varphi|^{2}+|x|^{2}|\varphi|^{2}\right)\right)^{A}
\end{aligned}
$$


Let

$$
\begin{gathered}
b:=\frac{C(p, \alpha, N)}{p}\left\|\varphi_{0}\right\|_{L^{2}}^{2 B}=\frac{1}{B}\left(\frac{B}{A}\right)^{A}\|w\|_{L^{2}}^{2-2 p}\left\|\varphi_{0}\right\|_{L^{2}}^{2 B}, \\
\theta=A=\frac{N(p-2)+\alpha}{2}>1 \quad \text { and } \\
\Phi(t)=\int\left(|\nabla \varphi(t)|^{2}+|x|^{2}|\varphi(t)|^{2}\right) .
\end{gathered}
$$

Obviously $\Phi(0)=a$. At the same time, we define $f(y)=a-y+b y^{\theta}$. Then (5.2) implies that

$$
0<a-y+b y^{\theta}, \quad \text { where } \quad y=\Phi(t) .
$$

Denote

$$
y_{*}=(b \theta)^{-\frac{1}{\theta-1}}, \quad b_{*}=\frac{\theta-1}{\theta} y_{*} .
$$

Then $b_{*}<y_{*}$. By direct computation and the exact value of $C(p, \alpha, N)$ we have that

$$
y_{*}=\frac{A}{B}\|w\|_{L^{2}}^{\frac{2(p-1)}{A-1}}\left\|\varphi_{0}\right\|_{L^{2}}^{-\frac{2 B}{A-1}}
$$

and

$$
b_{*}=\frac{A-1}{B}\|w\|_{L^{2}}^{\frac{2(p-1)}{A-1}}\left\|\varphi_{0}\right\|_{L^{2}}^{-\frac{2 B}{A-1}} .
$$

Moreover, using $\left\|\varphi_{0}\right\|_{L^{2}} \leq V\left(\left\|\nabla \varphi_{0}\right\|_{L^{2}}^{2}+\left\|x \varphi_{0}\right\|_{L^{2}}^{2}\right)=V(a)$, we know that

$$
\left\|\varphi_{0}\right\|_{L^{2}} \leq\left(\frac{A-1}{B}\right)^{\frac{A-1}{2 B}}\|w\|_{L^{2}}^{\frac{p-1}{B}} a^{-\frac{A-1}{2 B}}
$$

which implies that

$$
a \leq \frac{A-1}{B}\|w\|_{L^{2}}^{\frac{2(p-1)}{A-1}}\left\|\varphi_{0}\right\|_{L^{2}}^{-\frac{2 B}{A-1}}
$$

because of $2+(2-\alpha) / N<p<2 *(\alpha)$.

Now using (5.4), (5.5) and Lemma 5.1, we obtain that $\Phi(t)<y_{*}$ for any $t \in[0, T)$. It follows from $\int|\varphi|^{2} \equiv \int\left|\varphi_{0}\right|^{2}$ that $\|\varphi(t)\|_{\Sigma}^{2}$ is bounded from above uniformly with respect to $t \in[0, T)$. In other words, the solutions of equations (1.2)-(1.3) with $\varphi_{0}$ satisfying (5.1) exist globally in $t \in[0,+\infty)$.

Since $\Phi(t)<y_{*}$ for any $t \in[0, T)$, we obtain that

$$
\left\|\varphi_{0}\right\|_{L^{2}}^{\frac{2 B}{A-1}}\left(\|\nabla \varphi(t)\|_{L^{2}}^{2}+\|x \varphi(t)\|_{L^{2}}^{2}\right)<\frac{A}{B}\|w\|_{L^{2}}^{\frac{2(p-1)}{A-1}} .
$$

Next, for the solutions obtained in the above, we give an explicit upper bound on 
$\|\varphi(t)\|_{\Sigma}^{2}$. First we have that

$$
\begin{aligned}
E\left(\varphi_{0}\right)= & E(\varphi)=\frac{1}{2} \int\left(|\nabla \varphi|^{2}+|x|^{2}|\varphi|^{2}\right)-\frac{1}{2 p} \int_{\mathbb{R}^{N} \times \mathbb{R}^{N}} \frac{|\varphi(x)|^{p}|\varphi(y)|^{p}}{|x-y|^{\alpha}} d x d y \\
\geq & \frac{1}{2} \int\left(|\nabla \varphi|^{2}+|x|^{2}|\varphi|^{2}\right)-\frac{C(p, \alpha, N)}{2 p}\|\varphi\|_{L^{2}}^{2 B}\|\nabla \varphi\|_{L^{2}}^{2 A} \\
\geq & \frac{1}{2} \int\left(|\nabla \varphi|^{2}+|x|^{2}|\varphi|^{2}\right)-\frac{C(p, \alpha, N)}{2 p}\|\varphi\|_{L^{2}}^{2 B}\left(\int\left(|\nabla \varphi|^{2}+|x|^{2}|\varphi|^{2}\right)\right)^{A} \\
= & \frac{1}{2} \int\left(|\nabla \varphi|^{2}+|x|^{2}|\varphi|^{2}\right)\left[1-\frac{C(p, \alpha, N)}{p}\|\varphi\|_{L^{2}}^{2 B}\right. \\
& \left.\quad \times\left(\int\left(|\nabla \varphi|^{2}+|x|^{2}|\varphi|^{2}\right)\right)^{A-1}\right] \\
= & \frac{1}{2} \int\left(|\nabla \varphi|^{2}+|x|^{2}|\varphi|^{2}\right)\left(1-\frac{1}{A}\left[\left(\frac{A C(p, \alpha, N)}{p}\left\|\varphi_{0}\right\|_{L^{2}}^{2 B}\right)^{-\frac{1}{A-1}}\right.\right. \\
& \left.\left.\quad \times\left(\int\left(|\nabla \varphi|^{2}+|x|^{2}|\varphi|^{2}\right)\right)^{-1}\right]^{1-A}\right) .
\end{aligned}
$$

Since $\Phi(t)<y_{*}$, we have that

$$
\left(\frac{A C(p, \alpha, N)}{p}\left\|\varphi_{0}\right\|_{L^{2}}^{2 B}\right)^{-\frac{1}{A-1}}\left(\int\left(|\nabla \varphi|^{2}+|x|^{2}|\varphi|^{2}\right)\right)^{-1}>1
$$

by using the exact value of $C(p, \alpha, N)$ obtained in Theorem 2.7. Therefore

$$
\left[\left(\frac{A C(p, \alpha, N)}{p}\left\|\varphi_{0}\right\|_{L^{2}}^{2 B}\right)^{-\frac{1}{A-1}}\left(\int\left(|\nabla \varphi|^{2}+|x|^{2}|\varphi|^{2}\right)\right)^{-1}\right]^{1-A}<1
$$

because of $2+(2-\alpha) / N<p<\frac{2 N-\alpha}{N-2}$. It follows that

$$
E\left(\varphi_{0}\right) \geq \frac{1}{2} \int\left(|\nabla \varphi|^{2}+|x|^{2}|\varphi|^{2}\right)\left(1-\frac{1}{A}\right),
$$

which yields that

$$
\int\left(|\nabla \varphi|^{2}+|x|^{2}|\varphi|^{2}\right) \leq \frac{2 N(p-2)+2 \alpha}{N(p-2)+\alpha-2} E\left(\varphi_{0}\right) .
$$

Therefore

$$
\|\varphi(t)\|_{\Sigma}^{2} \leq \frac{2 N(p-2)+2 \alpha}{N(p-2)+\alpha-2} E\left(\varphi_{0}\right)+\left\|\varphi_{0}\right\|_{L^{2}}^{2} .
$$

The proof is complete.

REmark 5.4. By Lemma 5.2, we get that equations (1.2)-(1.3) possesses global solutions for a large class of initial data whose norm can be as large as we want. On the other hand, from the definition of $V(\lambda)$ and Theorem 5.3 we know that $V(\lambda) \rightarrow\|w\|_{L^{2}}$ as $p \rightarrow 2+(2-\alpha) / N$. So we obtain the sharp condition for global existence in the case of initial data $\left\|\varphi_{0}\right\|_{L^{2}}<\|w\|_{L^{2}}$, which coincides with Theorem 4.1. In the case of critical nonlinearity $p=2+(2-\alpha) / N$, the condition (4.1) is sharp. However, we do not know whether or not the condition (5.1) is sharp in the case of supercritical nonlinearity $2+(2-\alpha) / N<p<2^{*}(\alpha)$. 
Appendix. In this appendix, we outline the proof of Proposition 3.1 by using similar arguments [20]. Consider the Schrödinger operator in $\mathbb{R}^{N}$,

$$
A=-\Delta+|x|^{2} .
$$

Let $S(t)$ be the propagator of $A$ and $k(t, x, y)$ be its Schwartz kernel. It is known from [20] that

$$
k(t, x, y)=\left(\frac{-i}{2 \pi \sin t}\right)^{N / 2} e^{i\left\{\cos t\left(|x|^{2}+|y|^{2}\right)-2 x \cdot y\right\} / 2 \sin t} .
$$

Oh [20] proved the following proposition

Proposition A.1 ([20]). For any $q \geq 2$ and $0<|t| \leq T_{0}, S(t)$ is a bounded operator from $L^{q^{\prime}}$ to $L^{q}, 1 / q+1 / q^{\prime}=1$, and the map $t \rightarrow S(t)$ is strongly continuous to $B\left(L^{q^{\prime}}, L^{q}\right)$. Moreover, for $0<|t| \leq T_{0}$, we have

$$
\|S(t) u\|_{q} \leq\left(\frac{2 \pi|t|}{C_{1}}\right)^{N / q-N / 2}\|u\|_{q^{\prime}}
$$

where $C_{1}=\sup _{x, y,|t| \leq T_{0}}\left|\frac{t}{\sin t}\right|^{1 / 2}$.

Definition A.2 (admissible pair). The pair $(r, q)$ is admissible if $2 / r=N(1 / 2-$ $1 / q), 2 \leq q \leq \infty$ for $N=1,2 \leq q<\infty$ for $N=2$, and $2 \leq q<2 N /(N-2)$ for $N \geq 3$.

Lemma A.3. Let $(r, q)$ be any admissible pair.

(I) For any $0<T \leq T_{0}$ and $\psi \in L^{2}\left(R^{N}\right)$, we have $S(t) \psi \in L^{r}\left(-T, T ; L^{q}\right)$, and there exists a constant $C$ such that

$$
\|S(t) \psi\|_{L^{r}\left(-T, T ; L^{q}\right)} \leq C\|\psi\|_{L^{2}} .
$$

(II) For any $0<T \leq T_{0}$ and $g \in L^{r^{\prime}}\left(-T, T ; L^{q^{\prime}}\left(R^{N}\right)\right)$, we have $\int_{0}^{t} S(t-\tau) g(\tau) d \tau \in$ $L^{r}\left(-T, T ; L^{q}\right) \cap C\left([-T, T] ; L^{2}\right)$, and there exists a constant $C$ such that

$$
\begin{aligned}
& \left\|\int_{0}^{t} S(t-\tau) g(\tau) d \tau\right\|_{L^{r}\left(-T, T ; L^{q}\right)} \leq C\|g\|_{L^{r^{\prime}}\left(-T, T ; L^{q^{\prime}}\right)}, \\
& \left\|\int_{0}^{t} S(t-\tau) g(\tau) d \tau\right\|_{C\left([-T, T] ; L^{2}\right)} \leq C\|g\|_{L^{r^{\prime}}\left(-T, T ; L^{q^{\prime}}\right)} .
\end{aligned}
$$

Proof. For any $\psi \in L^{2}\left(R^{N}\right)$ and $f \in L^{r^{\prime}}\left(-T, T ; L^{q^{\prime}}\left(R^{N}\right)\right)$, we obtain

$$
\begin{aligned}
&\left|\int_{-T}^{T} \int_{R^{N}} S(t) \psi \bar{f}(t) d x d t\right|=\left|\int_{-T}^{T} \int_{R^{N}} \psi \bar{S}(-t) \bar{f}(t) d x d t\right| \\
& \leq\|\psi\|_{L_{x}^{2}}\left\|\int_{-T}^{T} S(-t) f(t) d t\right\|_{L_{x}^{2}}, \\
&\left\|\int_{-T}^{T} S(-t) f(t) d t\right\|_{L_{x}^{2}}^{2}=\int_{-T}^{T} \int_{R^{N}} f(t, x) \int_{-T}^{T} \bar{S}(t-\tau) \bar{f}(\tau) d \tau d x d t
\end{aligned}
$$




$$
\leq\|f\|_{L^{r^{\prime}}\left(-T, T ; L^{q^{\prime}}\right)}\left\|\int_{-T}^{T} S(t-\tau) f(\tau) d \tau\right\|_{L^{r}\left(-T, T ; L^{q}\right)} .
$$

By using equation (A.1) and Hardy-Littlewood-Sobolev inequality (see, e.g. [23, 117120]) we obtain

$$
\begin{gathered}
\left\|\int_{-T}^{T} S(t-\tau) f(\tau) d \tau\right\|_{L^{r}\left(-T, T ; L^{q}\right)} \leq C\left\|\int_{-T}^{T}\right\| t-\left.\tau\right|^{N / q-N / 2} \mid f(\tau)\left\|_{L_{x}^{q^{\prime}}} d \tau\right\|_{L^{r}(-T, T)} \\
\leq C\|f\|_{L^{r^{\prime}}\left(-T, T ; L^{q^{\prime}}\right)}
\end{gathered}
$$

From equations (A.5)-(A.7) we have equation (A.2).

For any $f \in L^{r^{\prime}}\left(-T, T ; L^{q^{\prime}}\left(R^{N}\right)\right)$, by using equation (A.2) and equation (A.7) we obtain

$$
\begin{gathered}
\quad\left|\int_{-T}^{T} \int_{R^{N}} \int_{0}^{t} S(t-\tau) g(\tau) d \tau \bar{f}(t, x) d x d t\right| \\
=\left|\int_{0}^{t} \int_{R^{N}} g(\tau, x) \int_{-T}^{T} \bar{S}(\tau-t) \bar{f}(t) d t d x d \tau\right| \\
\leq\|g\|_{L^{r^{\prime}}\left(-T, T ; L^{q^{\prime}}\right)}\left\|\int_{-T}^{T} S(t-\tau) f(\tau) d \tau\right\|_{L^{r}\left(-T, T ; L^{q}\right)} \\
\leq C\|g\|_{L^{r^{\prime}}\left(-T, T ; L^{q^{\prime}}\right)}\|f\|_{L^{r^{\prime}}\left(-T, T ; L^{q^{\prime}}\right)} \\
\left\|\int_{0}^{t} S(t-\tau) g(\tau) d \tau\right\|_{L^{r}\left(-T, T ; L^{q}\right)} \leq C\|g\|_{L^{r^{\prime}}\left(-T, T ; L^{q^{\prime}}\right)}, \\
\left\|\int_{0}^{t} S(t-\tau) g(\tau) d \tau\right\|_{L_{x}^{2}}^{2}=\int_{0}^{t} \int_{R^{N}} g(\tau, x) \bar{S}(\tau-t) \int_{0}^{t} \bar{S}(t-s) \bar{g}(\tau) d s d x d \tau \\
\leq\|g\|_{L_{\tau}^{r^{\prime}}\left(-T, T ; L^{q^{\prime}}\right)}\left\|S(\tau-t) \int_{0}^{t} S(t-s) g(s) d s\right\|_{L_{\tau}^{r}\left(-T, T ; L^{q}\right)} \\
\leq C\|g\|_{L_{\tau}^{r^{\prime}}\left(-T, T ; L^{q^{\prime}}\right)}\left\|\int_{0}^{t} S(t-s) g(s) d s\right\|_{L_{x}^{2}}, \\
\left\|\int_{0}^{t} S(t-\tau) g(\tau) d \tau\right\|_{C\left([-T, T] ; L^{2}\right)} \leq C\|g\|_{L^{r^{\prime}}\left(-T, T ; L^{q^{\prime}}\right)} .
\end{gathered}
$$

This completes the proof of Lemma A.3.

Now applying Lemma A.3, we can prove the local existence of solution for equations (1.2)-(1.3) and obtain some conservation laws with $\varphi_{0} \in \sum$. We consider the integral equation associated to equation (1.2)

$$
\varphi(t)=S(t) \varphi_{0}-i \int_{0}^{t} S(t-\tau) F(\varphi(\tau)) d \tau, \quad F(\psi):=\psi|\psi|^{p-2} \int_{R^{N}} \frac{|\psi(y)|^{p}}{|x-y|^{\alpha}} d y .
$$

For getting some conservation laws precise, we also consider regularized versions of equation (A.10), as in [20, 15]

$$
\varphi(t)=S(t) h *\left(g \varphi_{0}\right)-i \int_{0}^{t} S(t-\tau) h *\{g F(h * \varphi(\tau))\} d \tau,
$$


where we let $h$ belong to the Schwarz space and be an even function with $\|h\|_{L^{1}}=1$, and $g$ be a $C^{\infty}$ cut-off function with compact support. From now on, we denote $\phi=$ $(g, h)$ for notational convenience. Let $\delta$ denote the Dirac delta function. If $\phi=(1, \delta)$, then equation equation (A.11) is equivalent to equation equation (A.10).

Let

$$
\frac{1}{\rho}= \begin{cases}\frac{2 N-\alpha+2 p-2}{2 p N}, & N \geq 3 \\ \frac{1}{2}\left(\frac{N-\alpha}{2 N}+\max \left\{\frac{1}{2}-\frac{1}{p N}, \frac{2 N-\alpha}{2 p N}\right\}\right), & N=1,2\end{cases}
$$

and $2 / \gamma=N(1 / 2-1 / \rho)$. It is obvious that $(\gamma, \rho)$ is an admissible pair.

Lemma A.4. Let $0<\alpha<N, 2 \leq p<2^{*}(\alpha)$, and $\rho$ be defined in equation (A.12). For any $\psi \in H_{\rho}^{1}\left(R^{N}\right)$, we have

$$
\|F(\psi)\|_{H_{\rho^{\prime}}^{1}} \leq C\|\psi\|_{H_{\rho}^{1}}^{2 p-1}
$$

Proof. By using the Hölder inequality, we obtain

$$
\begin{aligned}
\|F(\psi)\|_{H_{\rho^{\prime}}^{1} \leq} & C\|\psi\|_{H_{\rho}^{1}}\|\psi\|_{L^{\rho^{*}}}^{p-2}\left\||\psi|^{p} *|y|^{-\alpha}\right\|_{L^{q_{1}}} \\
& +C\|\psi\|_{L^{\rho *}}^{p-1}\left\|\left\{|\psi|^{p-2}\left(\bar{\psi} \partial_{y} \psi+\psi \partial_{y} \bar{\psi}\right)\right\} *|y|^{-\alpha}\right\|_{L^{q_{2}}},
\end{aligned}
$$

where

$$
\frac{1}{\rho_{*}}=\frac{1}{2 p-2}\left(1-\frac{2}{\rho}+\frac{N-\alpha}{N}\right), \frac{1}{q_{1}}=\frac{p}{\rho_{*}}-\frac{N-\alpha}{N}, \frac{1}{q_{2}}=\frac{1}{\rho}+\frac{p-1}{\rho_{*}}-\frac{N-\alpha}{N} .
$$

By the Hardy-Littlewood-Sobolev inequality (see, e.g. [23, 117-120]), we obtain

$$
\|F(\psi)\|_{H_{\rho^{\prime}}^{1}} \leq C\|\psi\|_{H_{\rho}^{1}}\|\psi\|_{L^{\rho_{*}}}^{2 p-2} .
$$

From (A.12), we have

$$
\frac{1}{\rho} \geq \frac{1}{\rho_{*}} \geq \frac{1}{\rho}-\frac{1}{N}
$$

By applying the Sobolev imbedding theorem and equation (A.15), we obtain equation (A.13).

Lemma A.5. Let $0<T \leq T_{0}, 0<\alpha<N, 2 \leq p<(2 N-\alpha) /(N-2)$, $\rho$ be defined in equation (A.12), and $(\gamma, \rho)$ be the admissible pair. For any $u, v \in L^{\gamma}\left(-T, T ; H_{\rho}^{1}\left(R^{N}\right)\right)$, we have

$$
\begin{gathered}
\left\|\int_{0}^{t} S(t-\tau) h *\{g F(h * u)-g F(h * v)\} d \tau\right\|_{L^{\gamma}\left(-T, T ; L^{\rho}\right)} \\
\leq C T^{1-2 p / \gamma}\left\{\|u\|_{L^{\gamma}\left(-T, T ; H_{\rho}^{1}\right)}^{2 p-2}+\|v\|_{L^{\gamma}\left(-T, T ; H_{\rho}^{1}\right)}^{2 p-2}\right\}\|u-v\|_{L^{\gamma}\left(-T, T ; L^{\rho}\right)}, \\
\left\|\int_{0}^{t} S(t-\tau) h *\{g F(h * u)\} d \tau\right\|_{L^{\gamma}\left(-T, T ; H_{\rho}^{1}\right)} \leq C T^{1-2 p / \gamma}\|u\|_{L^{\gamma}\left(-T, T ; H_{\rho}^{1}\right)}^{2 p-1},
\end{gathered}
$$


where the constant $C$ is independent of $\phi=(g, h)$.

Proof. By using equation (A.3), the Hölder inequality, and the Sobolev imbedding theorem, we have

$$
\begin{aligned}
& \left\|\int_{0}^{t} S(t-\tau) h *\{g F(h * u)-g F(h * v)\} d \tau\right\|_{L^{\gamma}\left(-T, T ; L^{\rho}\right)} \\
& \leq C\|F(h * u)-F(h * v)\|_{L \gamma^{\prime}\left(-T, T ; H_{\rho^{\prime}}^{1}\right)} \\
& \leq C\left(\int _ { - T } ^ { T } \left\{\|(u-v)(t)\|_{L^{\rho}}^{\gamma^{\prime}}\left(\|u(t)\|_{L^{\rho_{*}}}^{(p-2) \gamma^{\prime}}+\|v(t)\|_{L^{\rho *}}^{(p-2) \gamma^{\prime}}\right)\left\||h * u|^{p} *|y|^{-\alpha}\right\|_{L^{q_{1}}}^{\gamma^{\prime}}\right.\right. \\
& \left.\left.+\|v(t)\|_{L^{\rho *}}^{(p-1) \gamma^{\prime}}\left\|\left(|h * u|^{p}-|h * v|^{p}\right) *|y|^{-\alpha}\right\|_{L^{q_{2}}}^{\gamma^{\prime}}\right\} d t\right)^{1 / \gamma^{\prime}} \\
& \leq C\left(\int_{-T}^{T}\|(u-v)(t)\|_{L^{\rho}}^{\gamma^{\prime}}\left(\|u(t)\|_{L^{\rho *}}^{(2 p-2) \gamma^{\prime}}+\|v(t)\|_{L^{\rho *}}^{(2 p-2) \gamma^{\prime}}\right) d t\right)^{1 / \gamma^{\prime}} \\
& \leq C T^{1-2 p / \gamma}\left\{\|u\|_{L^{\gamma}\left(-T, T ; H_{\rho}^{1}\right)}^{2 p-2}+\|v\|_{L^{\gamma}\left(-T, T ; H_{\rho}^{1}\right)}^{2 p-2}\right\}\|u-v\|_{L^{\gamma}\left(-T, T ; L^{\rho}\right)},
\end{aligned}
$$

where $\rho_{*}, q_{1}$ and $q_{2}$ are defined in equation (A.14), constant $C$ is independent of $\phi=(g, h)$.

From Lemmas A.3 and A.4, we obtain that

$$
\begin{gathered}
\left\|\int_{0}^{t} S(t-\tau) h *\{g F(h * u)\} d \tau\right\|_{L^{\gamma}\left(-T, T ; H_{\rho}^{1}\right)} \leq C\|F(h * u)\|_{L^{\gamma^{\prime}}\left(-T, T ; H_{\rho^{\prime}}^{1}\right)} \\
\quad \leq C\left(\int_{-T}^{T}\|u(t)\|_{H_{\rho}^{1}}^{(2 p-1) \gamma^{\prime}} d t\right)^{1 / \gamma^{\prime}} \leq C T^{1-2 p / \gamma}\|u\|_{L^{\gamma}\left(-T, T ; H_{\rho}^{1}\right)}^{2 p-1},
\end{gathered}
$$

where the constant $C$ does not depend on $\phi=(g, h)$.

Lemma A.6. Let $0<\alpha<N$ and $2 \leq p<2^{*}(\alpha)$. For any $\varphi_{0} \in H^{1}\left(R^{N}\right)$, there exists a $0<T=T\left(\left\|\varphi_{0}\right\|_{H^{1}}\right) \leq T_{0}$ and a unique solution $\varphi$ of equations (A.11) and (A.12) such that $\varphi \in C\left([-T, T] ; H^{1}\left(R^{n}\right)\right) \cap L^{\gamma}\left(-T, T ; H_{\rho}^{1}\left(R^{N}\right)\right)$, where $T$ does not depend on $\phi, \rho$ is defined in equation (A.12), and $(\gamma, \rho)$ is admissible pair.

Proof. Let constants $T>0$ and $R_{0}>0$ be selected later, and

$$
E=E\left(T, R_{0}\right)=\left\{\varphi \mid \varphi \in L^{\gamma}\left(-T, T ; H_{\rho}^{1}\left(R^{N}\right)\right),\|\varphi\|_{L^{\gamma}\left(-T, T ; H_{\rho}^{1}\left(R^{N}\right)\right)} \leq R_{0}\right\} .
$$

Note that $E$ is never empty by Lemma A.3. Endowed with the metric

$$
\operatorname{dist}(u, v)=\|u-v\|_{L^{\gamma}\left(-T, T ; L^{\rho}\right)},
$$

$E$ is a complete metric space. Indeed, since $L^{\gamma}\left(-T, T ; H_{\rho}^{1}\left(R^{N}\right)\right)$ is reflexive, the closed ball of radius $R_{0}$ is weakly compact. We wish to find conditions on $T$ and $R_{0}$ such that the map $M: \varphi \rightarrow M \varphi$ given by

$$
\begin{gathered}
M \varphi=S(t) h *\left(g \varphi_{0}\right)-i \int_{0}^{t} S(t-\tau) h *\{g F(h * \varphi(\tau))\} d \tau \text { and } \\
F(\psi):=\psi|\psi|^{p-2} \int_{R^{N}} \frac{|\psi(y)|^{p}}{|x-y|^{\alpha}} d y
\end{gathered}
$$


is a strict contraction on $E$. For any $\varphi \in E$, from Lem. A.3 and A.5 we have

$$
\|M \varphi\|_{L^{\gamma}\left(-T, T ; H_{\rho}^{1}\left(R^{N}\right)\right)} \leq C\left\|\varphi_{0}\right\|_{H^{1}}+C T^{1-2 p / \gamma}\|\varphi\|_{L^{\gamma}\left(-T, T ; H_{\rho}^{1}\left(R^{N}\right)\right)}^{2 p-1},
$$

where the constant $C$ is independent of $\phi=(g, h)$. Let us take $R_{0}=2 C\left\|\varphi_{0}\right\|_{H^{1}}$. Note that $1-2 p / \gamma>0$. Thus there exists $T>0$, which does not depend on $\phi=(g, h)$, such that for any $\varphi \in E$ we have

$$
C T^{1-2 p / \gamma}\|\varphi\|_{L^{\gamma}\left(-T, T ; H_{\rho}^{1}\left(R^{N}\right)\right)}^{2 p-1} \leq R_{0} / 2 .
$$

This implies that $M: E \rightarrow E$. For any $u, v \in E$, it follows from Lemma A.5 that

$$
\begin{aligned}
& \|M u-M v\|_{L^{\gamma}\left(-T, T ; L^{\rho}\right)} \\
& \leq C T^{1-2 p / \gamma}\left\{\|u\|_{L^{\gamma}\left(-T, T ; H_{\rho}^{1}\right)}^{2 p-2}+\|v\|_{L^{\gamma}\left(-T, T ; H_{\rho}^{1}\right.}^{2 p-2}\right\}\|u-v\|_{L^{\gamma}\left(-T, T ; L^{\rho}\right)},
\end{aligned}
$$

where the constant $C$ is independent of $\phi=(g, h)$. Then there exists $0<T=T\left(\varphi_{0} \|_{H^{1}}\right)$, which does not depend on $\phi=(g, h)$, such that

$$
\|M u-M v\|_{L^{\gamma}\left(-T, T ; L^{\rho}\right)} \leq \frac{1}{2}\|u-v\|_{L^{\gamma}\left(-T, T ; L^{\rho}\right)} .
$$

So $M: E \rightarrow E$ is strictly contractive. There exists a unique fixed point $\varphi \in E$. From Lemma A.3, $\varphi \in C\left([-T, T] ; H^{1}\left(R^{N}\right)\right)$. Lemma A.6 is proved.

Lemma A.7. Let $0<\alpha<N, 2 \leq p<2^{*}(\alpha), \varphi_{0} \in \sum$, and $\varphi \in C\left([-T, T] ; H^{1}\left(R^{N}\right)\right)$ be the solution of equation (A.12). Then $\varphi \in C\left([-T, T] ; \sum\right)$ and for all $t \in[-T, T], \varphi$ satisfies the equalities

$$
\begin{gathered}
\int_{\mathbb{R}^{N}}|\varphi(t)|^{2} d x=\int_{\mathbb{R}^{N}}\left|\varphi_{0}\right|^{2} d x, \\
E(\varphi(t))=E\left(\varphi_{0}\right) .
\end{gathered}
$$

Proof. Following the proof of Theorem 4.7 in [20], we can prove this lemma.

\section{Proof of Proposition 3.1.}

Proof. Applying Lemma A.6 and Lemma A.7, we obtain Proposition 3.1.

Acknowledgement. The authors greatly appreciate the unknown referees for their valuable comments and suggestions.

\section{REFERENCES}

[1] G. Baym and C.J. Pethick, Ground state properties of magnetically trapped Bosecondensed rubidium gas, Phys. Rev. Lett., 76, 6-9, 1996.

[2] P. Bégout, Necessary conditions and sufficient conditions for global existence in the nonlinear Schrödinger equation, Adv. Math. Sci. Appl., 12, 817-829, 2002.

[3] H.J. Brascamp, E.H. Lieb and J.M. Luttinger, A general rearrangment inequality for multiple integrals, J. Funct. Anal., 17, 227-237, 1974.

[4] A. Burchard and Y. Guo, Compactness via symmetriztion, J. Funct. Anal., 214, 40-73, 2004. 
[5] L. Caffarelli, R. Kohn and L. Nirenberg, First order interpolation inequalities with weights, Compositio Math., 53, 259-275, 1984.

[6] Y. Cao, Z.H. Musslimani and E.S. Titi, Nonlinear Schrödinger -Helmholtz equation as numerical regularization of the nonlinear Schrödinger equation, Nonlinearity, 21, 879-898, 2008.

[7] T. Cazenave, An Introduction to Nonlinear Schrödinger Equations, Textos de Metodos Matematicos, Rio de Janeiro, 22, 1989.

[8] G. Chen and J. Zhang, Remarks on global esistence for the supercritical nonlinear Schrödinger equation with a harmonic potential, J. Math. Anal. Appl., 320, 591-598, 2006.

[9] J. Chen and B. Guo, Strong instability of standing waves for a nonlocal Schrödinger equation, Phys. D, 227, 142-148, 2007.

[10] B. Deconinck and J.N. Kutz, Singular instability of exact stationary solutions of the nonlocal Gross-Pitaevskii, e-print cond-mat/0208441.

[11] R. Fukuizumi, Stability and instability of standing waves for the nonlinear Schrödinger equation with harmonic potential, Discrete Contin. Dyn. Syst., 7, 525-544, 2001.

[12] D. Fujiwara, A construction of the fundamental solution for the Schrödinger equation, J. Analyse Math., 35, 41-96, 1979.

[13] D. Fujiwara, Remarks on convergence of the Feynmam path integrals, Duke Math. J., 47, 559-600, 1980.

[14] J.J. Garcia-Ripoll, V.V. Konotop, B. Malomed and V.M. Perez-Garcia, A quasi-local Gross-Pitaevskii for Bose-Einstein condensates, Math. Compt. Simulation, 62, 2130, 2003.

[15] J. Ginibre and G. Velo, On a class of nonlinear Schrödinger equation, I: the Cauchy problem, J. Funct. Anal., 32, 1-32, 1979.

[16] R.T. Glassey, On the blowing-up of solutions to the Cauchy problem for the nonlinear Schrödinger equation, J. Math. Phys., 18, 1794-1797, 1977.

[17] Y.S. Kivshar, T.J. Alexander and S.K. Turitsyn, Nonlinear modes of a macroscopic quantum oscillator, Phys. Lett. A, 278, 225-230, 2001.

[18] M. Kurth, On the existence of infinitely many modes of a nonlocal nonlinear Schrödinger equation related to Dispersion-Managed solitons, SIAM J. Math. Anal., 36, 967-985, 2004.

[19] E.H. Lieb and M. Loss, Analysis, AMS Graduate studies in Mathematics, 2nd Edition, Providence AMS, 13, 2001.

[20] Y.G. Oh, Cauchy problem and Ehrenfest's law of nonlinear Schrödinger equations with potentials, J. Diff. Equ., 81, 255-274, 1989.

[21] M. Reed and B. Simon, Methods of Modern Mathematical Physics, Vols. II, IV, Elsevier (Singapore) Pte Ltd., 2003.

[22] H.A. Rose and M.I. Weinstein, On the bound states of the nonlinear Schrödinger equation with linear potential, Phys. D., 30, 207-218, 1988.

[23] E.M. Stein, Singular Integrals and Differentiability Properties of Functions, Princeton University Press, 1970.

[24] M.I. Weinstein, Nonlinear Schrödinger equations and sharp interpolation estimates, Commun. Math. Phys., 87, 567-576, 1983.

[25] J. Zhang, Stability of attractive Bose-Einstein condensates, J. Stat. Phys., 102, 731-746, 2000 . 